\title{
FIXED POINTS OF LOCALLY FUZZY CONTRACTIVE SET-VALUED MAPPINGS IN FUZZY METRIC SPACES
}

\author{
SHIHUANG HONG, CHUNXIANG ZHAO AND YINGZI PENG
}

\author{
Institute of Applied Mathematics \\ Hangzhou Dianzi University \\ Hangzhou, 310018, People's Republic of China \\ E-mail:hongshh@hotmail.com, yingzip@hdu.edu.cn
}

\begin{abstract}
In this paper, we first introduce new notions of the locally fuzzy contraction of set-valued mappings and $\varepsilon$-chainable fuzzy metric space. Using these notions, we deal with some issues of fixed point theory involving the generalization of fuzzy contractive mappings introduced by other authors. We enlarge this class and establish fuzzy versions of some known fixed point theorems(such as the Nadler's set-valued contractive and Edelstein's locally contractive fixed point theorems). The results are supported by examples.
\end{abstract}

Key Words and Phrases: Locally fuzzy contractive mapping, $\varepsilon$-chainable fuzzy metric space, $f w$-distance, fixed point.

2010 Mathematics Subject Classification: 37C25, 47H10.

\section{REFERENCES}

[1] A. George, P. Veeramani, On some results in fuzzy metric spaces, Fuzzy Sets Syst., 64(1994), no. 3, 395-399.

[2] A. George, P. Veeramani, On some results of analysis for fuzzy metric spaces, Fuzzy Sets Syst., 90(1997), no. 3, 365-368.

[3] O. Hadžić, E. Pap, Fixed Point Theory in Probabilistic Metric Space, Kluwer Academic Publishers, Dordrecht, 2001.

[4] S.B. Nadler, Multi-valued contraction mappings, Pacific J. Math., 30(1969), no. 2, 475-488.

[5] T. Suzuki, A generalized Banach contraction principle that characterizes metric completeness, Proc. Amer. Math. Soc., 136(2008), no. 5, 1861-1869.

[6] D. Wei-Shih, New existence results and generalizations for coincidence points and fixed points without global completeness, Abstract Appl. Anal., 2013(2013).

[7] M. Edelstein, An extension of Banach's contraction principle, Proc. Amer. Math. Soc., 12(1961), no. $1,7-10$.

[8] O. Kada, T. Suzuki, W. Takahashi, Nonconvex minimization theorems and fixed point theorems in complete metric spaces, Math. Japon, 44(1996), no. 2, 381-391.

[9] T. Suzuki, W. Takahashi, Fixed point theorems and characterizations of metric completeness, Topol. Meth. Nonlinear Anal., 8(1996), no. 2, 371-382.

[10] M. Grabiec, Fixed points in fuzzy metric spaces, Fuzzy Sets Syst., 27(1989), 385-389.

[11] R. Vasuki, A common fixed point theorem in a fuzzy metric space, Fuzzy Sets Syst., 97(1998), no. $3,395-397$. 
[12] V. Gregori, A. Sapena, On fixed-point theorems in fuzzy metric spaces, Fuzzy Sets Syst., 125(2002), no. 2, 245-252.

[13] R. Vasuki, P. Veeramani, Fixed point theorems and Cauchy sequences in fuzzy metric spaces Fuzzy Sets Syst., 135(2003), no. 3, 415-417.

[14] D. Mihet, Fuzzy $\psi$-contractive mappings in non-Archimedean fuzzy metric spaces, Fuzzy Sets Syst., 159(2008), no. 6, 739-744.

[15] C. Vetro, Fixed points in weak non-Archimedean fuzzy metric spaces, Fuzzy Sets Syst., 162(2011), no. 1, 84-90.

[16] S. Wang, Answers to some open questions on fuzzy $\psi$-contractions in fuzzy metric spaces, Fuzzy Sets Syst., 222(2013), 115-119.

[17] S.H. Hong, Y.Z. Peng, Fixed points of fuzzy contractive set-valued mappings and fuzzy metric completeness, Fixed Point Theory Appl., 2013(2013), 276.

[18] X.H. Zhu, J.Z. Xiao, Note on "Coupled fixed point theorems for contractions in fuzzy metric spaces", Nonlinear Anal., 74(2011), no. 16, 5475-5479.

[19] J. Xiao, X. Zhu, X. Jin, Fixed point theorems for nonlinear contractions in Kaleva-Seikkala's type fuzzy metric spaces, Fuzzy Sets Syst., 200(2012), 65-83.

[20] A. Roldán, J. Moreno, C. Roldán, Tripled fixed point theorem in fuzzy metric spaces and applications, Fixed Point Theory Appl., 2013(2013), 29.

[21] D. Wardowski, Fuzzy contractive mappings and fixed points in fuzzy metric spaces, Fuzzy Sets Syst., 222(2013), 108-114.

[22] L. Ćirić, Some new results for Banach contractions and Edelstein contractive mappings on fuzzy metric spaces, Chaos Solitons Fractals, 42(2009), no. 1, 146-154.

[23] F. Gu, W. Shatanawi, Common fixed point for generalized weakly G-contraction mappings satisfying common (E.A) property in G-metric spaces, Fixed Point Theory Appl., 2013(2013), 309.

[24] C. Ionescu, S. Rezapour, M.E. Samei, Fixed points of some new contractions on intuitionistic fuzzy metric spaces, Fixed Point Theory Appl., 2013(2013), 168.

25] S.H. Hong, Fixed points for modified fuzzy $\psi$-contractive set-valued mappings in fuzzy metric spaces, Fixed Point Theory Appl., 2014(2014), 12.

26] F. Kiany, A. Amini-Harandi, Fixed point and endpoint theorems for set-valued fuzzy contraction maps in fuzzy metric spaces, Fixed Point Theory Appl., 2011(2011).

[27] M. Arshad, A. Shoaib, Fixed points of a multivalued mappings in fuzzy metric spaces, Proceedings of the World Congress on Engineering, 2012 Vol I WCE 2012, July 4-6, London, U.K., 2012.

[28] J. Rodríguez-Lôpez, S. Romaguera, The Hausdorff fuzzy metric on compact sets, Fuzzy Sets Syst., 147(2004), no. 2, 273-283.

[29] I. Kramosil, J. Michalek, Fuzzy metrics and statistical metric spaces, Kybernetika, 11(1975), no. 5, 326-334.

[30] V. Gregori, J. Minana, S. Morillas, Some questions in fuzzy metric spaces, Fuzzy Sets Syst., 204(2012), 71-85.

[31] V. Gregori, S. Morillas, A. Sapena, Examples of fuzzy metrics and applications, Fuzzy Sets Syst., 170(2011), no. 1, 95-111.

[32] H.K. Nashine, C. Vetro, W. Kumam, P. Kumam, Fixed point theorems for fuzzy mappings and applications to ordinary fuzzy differential equations, Advances Difference Eq., 2014(2014), 232.

[33] P. Salimi, C. Vetro, P. Vetro, Some new fixed point results innon-Archimedean fuzzy metric spaces, Nonlinear Anal. Modelling and Control, 18(2013), 344-358.

Received: December 17, 2014; Accepted: May 15, 2015. 